\title{
The mGluR2/3 Agonist LY379268 Induced Anti-Reinstatement Effects in Rats Exhibiting Addiction-like Behavior
}

\author{
Nazzareno Cannella*,', Briac Halbout', Stefanie Uhrig', Lionel Evrard', Mauro Corsi ${ }^{2}$, Corrado Corti $^{2}$, \\ Veronique Deroche-Gamonet ${ }^{3,4}$, Anita C Hansson' and Rainer Spanagel' \\ IInstitute of Psychopharmacology, Central Institute of Mental Health, Faculty of Medicine Mannheim, University of Heidelberg, Heidelberg, \\ Germany; ${ }^{2}$ Aptuit, Medicine Research Centre, Verona, Italy; ${ }^{3}$ INSERM, Neurocentre Magendie, Physiopathologie de la plasticité neuronale, \\ Bordeaux, France; ${ }^{4}$ Université de Bordeaux, Bordeaux, France
}

Medication development for cocaine-addicted patients is difficult, and many promising preclinical candidates have failed in clinical trials. One reason for the difficulty in translating preclinical findings to the human condition is that drug testing is typically conducted in behavioral procedures in which animals do not show addiction-like traits. Recently, a DSM-IV-based animal model has been developed that allows studying the transition to an addiction-like behavior. Changes in synaptic plasticity are involved in the transition to cocaine addiction. In particular, it has been shown that metabotropic glutamate receptor 2/3 (mGluR2/3)-mediated long-term depression is suppressed in the prelimbic cortex in addict-like rats. We therefore hypothesized that cocaine-seeking in addict-like rats could be treated with an mGluR2/3 agonist. Indeed, addict-like rats that were treated systemically with the mGluR2/3 agonist LY379268 (0, 0.3, and $3 \mathrm{mg} / \mathrm{kg}$ ) showed a pronounced reduction in cue-induced reinstatement of cocaine-seeking. In an attempt to dissect the role played by mGluR2 and mGluR3 in cue-induced reinstatement, we analyzed the mRNA expression patterns in several relevant brain areas but did not find any significant differences between cocaine addict-like and non-addict-like rats, suggesting that the behavioral differences observed are due to translational rather than transcriptional regulation. Another possibility to study the contributions of mGluR2 and mGluR3 in mediating addictive-like behavior is the use of knockout models. Because mGluR2 knockouts cannot be used in operant procedures due to motoric impairment, we only tested mGluR3 knockouts. These mice did not differ from controls in reinstatement, suggesting that mGluR2 receptors are critical in mediating addictive-like behavior.

Neuropsychopharmacology (2013) 38, 2048-2056; doi:I0.1038/npp.20 I3.106; published online 29 May 2013

Keywords: group II metabotropic glutamate receptor; transition to addiction; relapse; drug escalation; mGluR3 deficient mice; in situ hybridization

\section{INTRODUCTION}

Addiction is a psychiatric disorder characterized by the loss of control over drug intake and a high vulnerability to relapse despite prolonged abstinence. Addictive behavior is diagnosed only in a small proportion of drug users who shift from controlled drug intake to compulsive use. The main neurobiological pathways and mechanisms driving drug self-administration have been previously identified (Koob and Volkow, 2010; Noori et al, 2012); however, the specific neuroanatomical substrates and mechanisms causing the transition to addiction are largely unknown, as animal models attempting to mimic the transition to addiction-like behavior have been developed only recently (Ahmed and Koob, 1998; Belin et al, 2009; Deroche-

* Correspondence: Dr N Cannella, Institute of Psychopharmacology, Central Institute of Mental Health (ZI), J5, 68159 Mannheim, Germany, Tel: +49621 1703 6249, Fax: +49621 17036255 ,

E-mail: nazzareno.cannella@zi-mannheim.de

Received 8 January 2013; revised 22 April 2013; accepted 22 April 2013; accepted article preview online 29 April 2013
Gamonet et al, 2004; Pelloux et al, 2007; for review, see Sanchis-Segura and Spanagel, 2006; for an historical perspective, see Wikler and Pescor, 1967; and also Wolffgramm and Heyne, 1995).

Recent work has provided initial insight into the neurobiological mechanisms involved in the transition to an addiction-like behavior. Using a self-administration procedure in rats that has been introduced as a DSM-IVbased model of cocaine addiction (Deroche-Gamonet et al, 2004), it was reported that during the early phase of cocaine self-administration, when addiction-like behavior had not yet developed, subjects showed a suppression of NMDA-dependent long-term depression (LTD) in the nucleus accumbens (NAc). However, after prolonged self-administration, during which addiction-like behavior developed in a subset of animals, LTD was recovered in non-addict-like, but not in addict-like rats. Thus, the pathological state of addictive-like behavior appears to be characterized by a loss of synaptic plasticity-a phenomenon termed as anaplasticity (Kasanetz et al, 2010). Furthermore, a synaptic marker of addiction-like behavior was found in the prelimbic medial prefrontal cortex (PreL), 
in which metabotropic glutamate receptors $2 / 3$ (mGluR2/3)mediated LTD was impaired in addict-like, but not in non-addict-like subjects (Kasanetz et al, 2012). Altogether, it has been proposed that the transition to addiction-like behavior is mediated by a lack of rescaling of synaptic potentiation occurring hierarchically in the limbic forebrain; ie, a persistent loss of NMDA-LTD in the NAc leading ultimately to a dysfunction of mGluR $2 / 3$ signaling in the prefrontal cortex (Kasanetz et al, 2010, 2012).

The release of glutamate from the PreL into the NAc core has been identified as the final common pathway driving relapse to cocaine-seeking, and an alteration in glutamate homeostasis in the limbic forebrain has been implicated in addiction (Kalivas, 2009; Kalivas and Volkow, 2005). In addition, a history of cocaine intake causes a decrease in basal extracellular glutamate levels, leading to a hypoactivation of inhibitory presynaptic mGluR $2 / 3$ receptors. Consequently, upon exposure to drug cues or stress, the synaptic release of glutamate in the NAc core is no longer compensated for via presynaptic inhibitory mechanisms, causing a loss of control over cocaine-seeking (Kalivas, 2009). This suggests that mGluR $2 / 3$ activation by direct agonists or potentiators, or alternatively by re-establishing physiological levels of glutamate, may be a suitable treatment for relapse behavior. Indeed, activating the glial cystine-glutamate exchanger during extinction of cocaine-seeking with daily treatment of the cystine prodrug $\mathrm{N}$-acetylcysteine induced an enduring normalization of extra-synaptic glutamate levels in the NAc core, and blocked reinstatement of cocaine-seeking through restoration of the glutamate tone on mGluR2/3 (Baker et al, 2003; Moussawi et al, 2011). Furthermore, exogenous activation of mGluR2/3 with its agonist LY379268 has been demonstrated to block reinstatement of cocaine-seeking induced by cues (Baptista et al, 2004), a priming dose of cocaine (Peters and Kalivas, 2006), and stress (Martin-Fardon and Weiss, 2012), as well as to impair the incubation of cocaine craving (Lu et al, 2007). Importantly, the role of mGluR2/3 agonists on cocaine-related behaviors has, to date, been studied only in non-dependent animals that had limited exposure to cocaine (12-24 days).

Here, we present data on the role of mGluR $2 / 3$ in addictlike and non-addict-like cocaine-experienced rats using the aforementioned DSM-IV-based rat model. We first report on the effect of the mGluR2/3 agonist LY379268 in cue-induced reinstatement in addict-like $v s$ non-addict-like rats and then present quantitative gene expression data on mGluR2 and mGluR3 following withdrawal from cocaine self-administration. To further dissect the role of these two different metabotropic glutamate receptors, we present data on cocaine self-administration, drug escalation, and cue-induced reinstatement in mGluR3 knockout (mGluR3 - /-) mice (Corti et al, 2007). Motoric impairments preclude the use of mGluR2 knockouts for operant studies (Morishima et al, 2005).

\section{MATERIALS AND METHODS}

All experiments were conducted at the Central Institute of Mental Health in Mannheim (Germany). Experimental procedures were in accordance with the NIH ethical guidelines for the care and use of laboratory animals, and were approved by the local animal care committee (Regierungspräsidium Karlsruhe, Germany).

\section{Experiments in Rats}

Male Sprague-Dawley rats were used in experiments 1 and 2, in which we used the DSM-IV-based rat model of cocaine addiction published by Deroche-Gamonet et al (2004). A cocaine self-administration session was composed of three 40-min 'Drug-periods' in which cocaine was delivered contingent upon nose-pokes at $0.8 \mathrm{mg} / \mathrm{kg} /$ infusion under Fixed Ratio (FR) 5, associated with a discrete white cue light and followed by a 40-s Time-Out (TO) period separated by two 15-min 'No-Drug-periods' discriminated by distinct light contexts; a blue cue light was on during 'Drug-periods' (except during TO), while the box was entirely illuminated by the house light during the 'No-Drug-periods'. After 45 sessions, rats were tested on the following three addictionlike criteria: (A) motivation for cocaine-taking, measured in a progressive ratio session, during which the break point achieved was used to score motivation; (B) persistence of cocaine-seeking, during which the subject continued to nose-poke for cocaine despite the unavailability of the drug, scored as the number of nose-pokes in the active hole; and (C) resistance to punishment, during which the subject continued to nose-poke for cocaine despite aversive consequences (two electric foot-shocks paired with cocaine-seeking and infusion, $0.8 \mathrm{~mA}$ in experiment 1 and $0.2 \mathrm{~mA}$ in experiment 2), scored as percent of baseline active poking. A subject was considered as positive for one criterion if its score was above the 60th percentile of the population distribution. Thus, depending on the number of positive criteria met, a subject was assigned to one of the following groups: $0 \mathrm{crit}, 1 \mathrm{crit}, 2 \mathrm{crit}$, and $3 \mathrm{crit}$. Rats negative for all criteria (0crit) were characterized as non-addict-like, whereas rats positive for all criteria (3crit) were characterized as addict-like (details in Supplementary Information). These two groups were subsequently used for further experiments.

Addiction score. An addiction score, a dimensional index of cocaine use severity described in Supplementary Information, was also calculated for each animal.

Reinstatement of cocaine-seeking. Cue-induced reinstatement of cocaine-seeking was tested after 30 days of withdrawal in 0 crit and 3 crit rats, during a unique 2-h session in which cocaine was not available. This session consisted of two periods; one initial 1-h period of extinction, during which nose-pokes in either hole had no scheduled consequences, was followed by 1 -h during which the 'Drug-period' discriminative light (blue cue light) was illuminated and the white cue associated with cocaine delivery during training was presented contingently to nose-pokes into the active hole.

Experiment 1: Effect of LY379268 on Cue-Induced Reinstatement in Addict-like and Non-Addict-like Rats. The effect of systemic LY379268 (0, 0.3, and $3.0 \mathrm{mg} / \mathrm{kg}$ IP) on cue-induced reinstatement was tested in 3 crit, $(n=6)$ and 0crit $(\mathrm{n}=6)$ rats using a latin-square design with 
sessions repeated every third day (details in Supplementary Information).

Experiment 2: mGluR2 and mGluR3 Gene Expression Levels in Addict-like vs Non-Addict-like Rats. Rats were trained, characterized, and subjected to a cue-induced reinstatement session as described for experiment 1, except that rats did not receive LY379268 treatment. Subsequently, gene expression of mGluR2 and mGluR3 was investigated using quantitative in situ hybridization as described in Supplementary Information and previously (Hansson et al, 2003).

\section{Experiments in Mice}

Male mGluR3 - I - and littermate control mice (Corti et al, 2007) were used in mouse self-administration experiments. Methods are fully described in Supplementary Information.

Experiment 3: Short-access cocaine self-administration, extinction, and cue-induced reinstatement of cocaineseeking in mGluR3 - / - and control mice.

Experiment 4: Long-access escalation of cocaine self-administration in mGluR3-I- and control mice.

\section{Data Analysis}

Group scores for each addiction-like criterion (motivation for cocaine-taking, persistence of cocaine-seeking, resistance to punishment) and addiction score were analyzed by descriptive statistics (mean, standard error, and population distribution), but not by ANOVA, as being groups based on population extremes their differences are significant. For cue-induced reinstatement in rat and mice experiments, we used a combined within- (lever, time, dose) and betweensubject (group) ANOVA. In situ hybridization data were compared region-wise using one-way ANOVA. Overall analyses were followed by post hoc when appropriate. All data are expressed as mean values \pm SEM. Significance was set at $p<0.05$.

For detailed statistical description, see Supplementary Information.

\section{RESULTS}

\section{Experiment 1: Effect of LY379268 on Cue-Induced Reinstatement in Addict-like and Non-Addict-like Rats}

Following cocaine self-administration training, rats were individually scored and assigned to groups based on the number of positive criteria met $(0,1,2$, and $3 \mathrm{crit}$; Supplementary Figures S1-S4). Rats negative for all criteria (0crit) were characterized as non-addict-like, whereas rats positive for all criteria (3crit) were characterized as addictlike. The 3 crit group scored higher in each addiction-like criterion than the 0crit group (Figure 1a-c), as well as for the addiction score, a computation of the three addictionlike criteria (Figure 1d).

Following a 30-day withdrawal, we tested cue-induced reinstatement after either saline or LY379268 treatment in 0 crit and 3 crit rats ( $n=6$ /group). An analysis of patterns of nose-pokes during extinction under the control condition revealed that both groups enter the cue-reinstatement part of the session with a similar baseline and low responding on both of the holes (Supplementary Figure S5). An analysis of cue-induced reinstatement showed a significant effect between groups $(\mathrm{F}(1,10)=7.28 ; p<0.05)$, indicating a different response between 0 crit and 3 crit, within holes $(\mathrm{F}(1,10)=18.85 ; \quad p<0.01), \quad$ extinction/cue $\quad(\mathrm{F}(1,10)=$ $48.38 ; p<0.0001)$, and holes $\times$ extinction/cue interaction
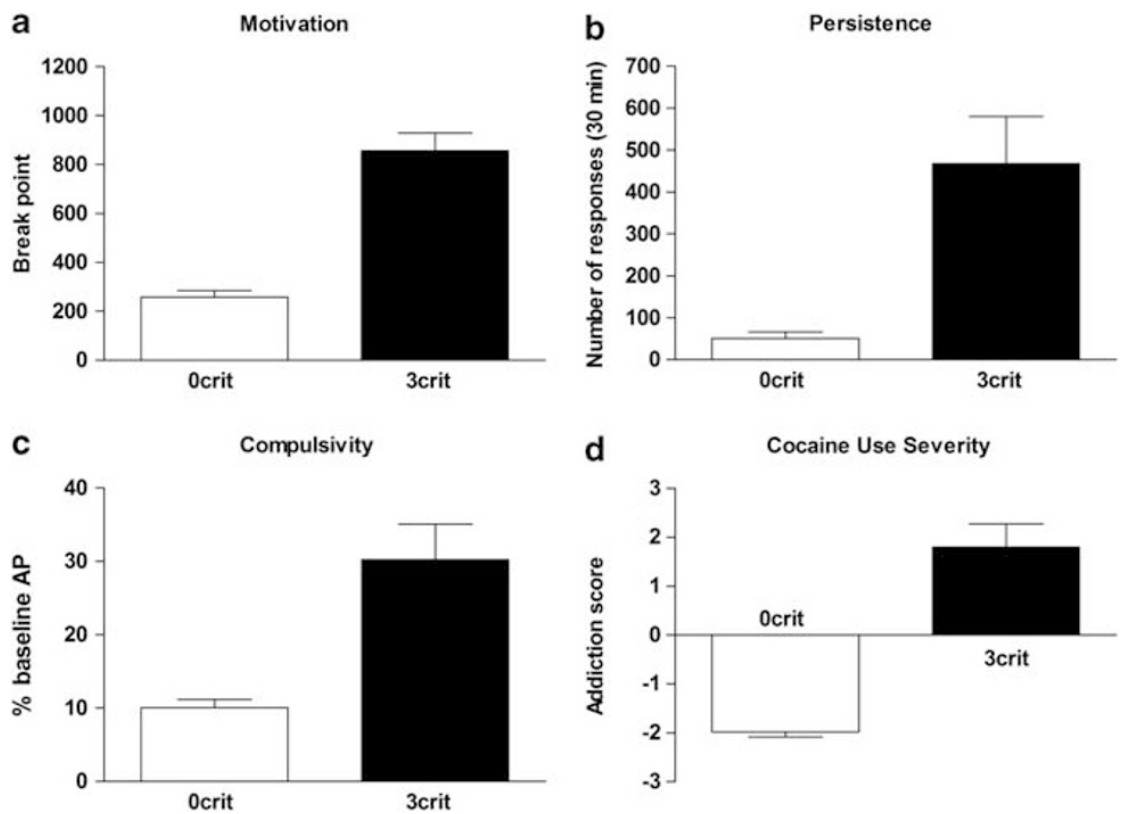

Figure I Experiment I: Rats characterized as addict-like (3crit, $n=6)$ showed higher response than non-addictive-like animals $(0 \mathrm{crit}, \mathrm{n}=13)$ in each criterion for addiction-like behavior. (a) Motivation for cocaine intake expressed as the break point during a progressive ratio session. (b) Persistence of cocaine-seeking expressed by active nose-pokes when the drug is signaled as unavailable. (c) Resistance to punishment expressed as \% of baseline active pokes (AP) when cocaine-seeking and taking are paired with a foot-shock punishment ( $0.8 \mathrm{~mA})$. (d) Addict-like rats had a higher cocaine-use severity as measured by computation of the three criteria in a single addiction score scale. Data are presented as mean \pm SEM. 
$(\mathrm{F}(1,10)=10.99 ; \quad p<0.01)$, indicating a discrimination between the two holes associated with the presentation of the cue. ANOVA also reported an overall effect of dose $(\mathrm{F}(2,20)=9.19 ; p<0.01)$ and dose $\times$ extinction/cue interaction $(\mathrm{F}(2,20)=10.90 ; p<0.001)$, but no dose $\times$ extinction/ cue $\times$ groups interaction $(\mathrm{F}(2,20)=0.53 ; p>0.05)$, indicating that LY379268 affected cue-induced reinstatement similarly in 0 crit and 3 crit rats. Post hoc analysis found no difference in extinction responding between 0 crit and 3 crit and within each group for any LY379268 dose, confirming that the two groups had a similar poke-baseline before exposure to the cue-reinstatement test, and that their baselines were not affected by LY379268 treatment (Figure 2a). When discriminative and contingent cocainecues were presented, active nose-poking was reinstated in both groups ( 0 crit $p<0.01,3$ crit $p<0.001$, vs extinction), and as expected was higher in 3 crit rats compared with 0 crit rats $(p<0.001)$. LY379268 reduced cue-induced reinstatement in both groups. In 0crit rats, both doses prevented reinstatement as no differences were observed within extinction and cue responding; in 3 crit rats, the dose of $0.3 \mathrm{mg} / \mathrm{kg}$ reduced but did not block cue-induced reinstatement ( $p<0.001 v s$ vehicle and extinction), whereas the dose of $3.0 \mathrm{mg} / \mathrm{kg}$ completely prevented cue-induced reinstatement, bringing the responses of 3 crit rats to extinction level and values similar to 0 crit (Figure $2 \mathrm{a}$ ). Inactive nose-poking was always low and at a similar level between 0 crit and 3 crit. The presentation of cocaine cues slightly increased inactive nose-poking in the 0crit group; however, this effect was not significant and was not a general feature of the group, due exclusively to a single subject (Figure 2a). These results demonstrate that LY379268 blocked cumulative cue-induced reinstatement in both 0 crit and 3 crit rats, abolishing a critical addiction-like feature; ie, increased cue-induced reinstatement in subjects characterized as addict-like.

Next, we explored the effect of LY379268 on the time pattern of cue-induced reinstatement in the two groups. In line with the cumulative analysis above, ANOVA of the time pattern of nose-pokes resulted in a dose $\times$ ext/cue $\times$ time interaction $(\mathrm{F}(4,40)=3.31 ; p<0.05)$ but no dose $\times$ ext/cue $\times$ time $\times$ group interaction $(\mathrm{F}(4,40)=0.57 ; p>0.05)$, indicating that LY379268 had a time-dependent effect on cue-induced reinstatement but this effect was similar between 0 crit and 3 crit; post hoc analysis confirmed that the two groups did not differ in pattern of responding during extinction and that dose effects of LY379268 were observable only during cue-induced reinstatement. However, browsing within post hoc data, we found a result worthy of mention, one hidden by the overall ANOVA; at the dose of $3.0 \mathrm{mg} / \mathrm{kg}$, LY379268 decreased the number of nose-pokes in 0crit rats only during the first time bin of cue-induced reinstatement, an effect that disappeared in the second and third time bins; conversely, in the 3 crit group, the anti-reinstatement effect of LY379268 lasted for the three cue-time bins (ie, with $3.0 \mathrm{mg} / \mathrm{kg}$ of LY379268 on board nose-poking was lower than control in each cue time bin). This result is unlikely to be a consequence of a higher number of nose-pokes produced by 3 crit respect to 0 crit under the control condition, as no significant differences were found in the second and third time bins between the groups (Figure 2b). Thus, LY379268 seems to have a longer lasting anti-reinstatement effect in 3 crit than in 0 crit rats. a

LY379268 on cumulative cue-induced reinstatement
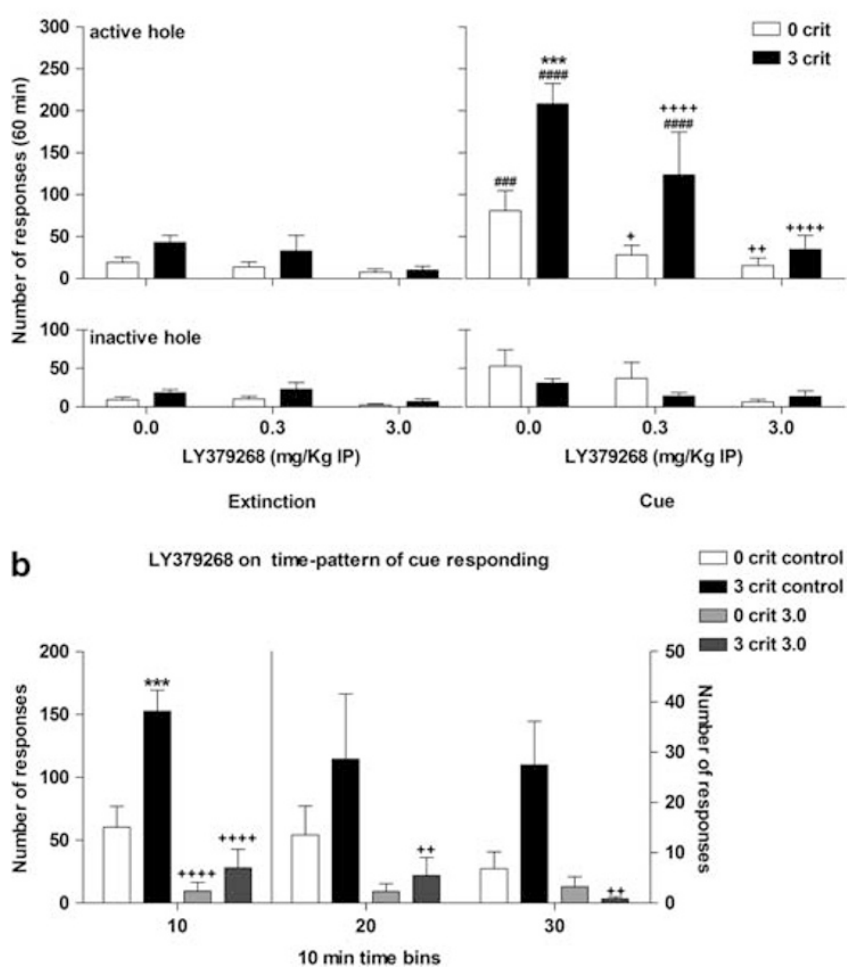

Figure 2 Experiment I: The effect of mGluR2/3 agonist LY379268 on cue-induced reinstatement of cocaine-seeking was tested in rats characterized as addict-like (3crit, $n=6$ ) and non-addict-like (0crit $n=6$ ). (a) Effect of LY379268 on cumulative cue-induced reinstatement. Immediately after extinction, cue-induced reinstatement initiated, during which rats poked to activate the discrete cocaine cue. As expected, 3crit rats responded significantly more than Ocrit. Both LY379268 doses prevented reinstatement of cocaine-seeking in 0crit rats. In the 3crit group, the highest dose abolished cue reinstatement while the lowest dose significantly decreased cocaine-seeking. (b) Time course of cue responding under control conditions and with the dose of $3.0 \mathrm{mg} / \mathrm{kg}$ during the three 10 -min time bins of cue-induced reinstatement. Given the drop in responding observed from the first to the second time bin, for figure clarity the first I0-min time bin is scaled by the left $y$ axis while the second and third are scaled by the right $y$ axis. For further figure clarity, time bins of extinction and the dose of $0.3 \mathrm{mg} / \mathrm{kg}$ are omitted. Data are presented as mean $\pm \mathrm{SEM}$; $* * * * 2<0.00$ I vs

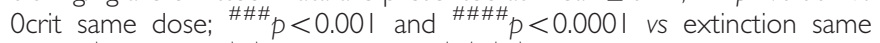
dose; ${ }^{+} p<0.05,{ }^{+}+p<0.01$, and ${ }^{++}++{ }^{+} p<0.0000$ I vs vehicle treatment same group.

\section{Experiment 2: mGluR2 and mGluR3 Gene Expression Levels in Addict-like vs Non-Addict-like Rats}

As for experiment 1, we assigned each rat to one of the 0 crit to 3 crit groups based on the number of positive criteria met (Supplementary Figures S6-S9), and we characterized 0crit $(n=7)$ rats as non-addict-like and $3 c$ rit $(n=5)$ as addictlike. Again, addict-like rats scored significantly higher than non-addict-like rats in each criterion as well as in the addiction score (Figure $3 \mathrm{~d}$ ). Two 3 crit and one 0 crit rats died during baseline re-training after the addiction-like criteria tests or withdrawal, and could not be used for cue-induced reinstatement and in situ analysis, thus only three addict-like and six non-addict-like rats performed the cue-induced reinstatement test. ANOVA of cue-induced reinstatement showed, as expected, an overall effect between groups $(\mathrm{F}(1,7)=10.37 ; p<0.05)$, within holes $(\mathrm{F}(1,7)=43.30 ; p<0.001)$ and extinction/cue condition 

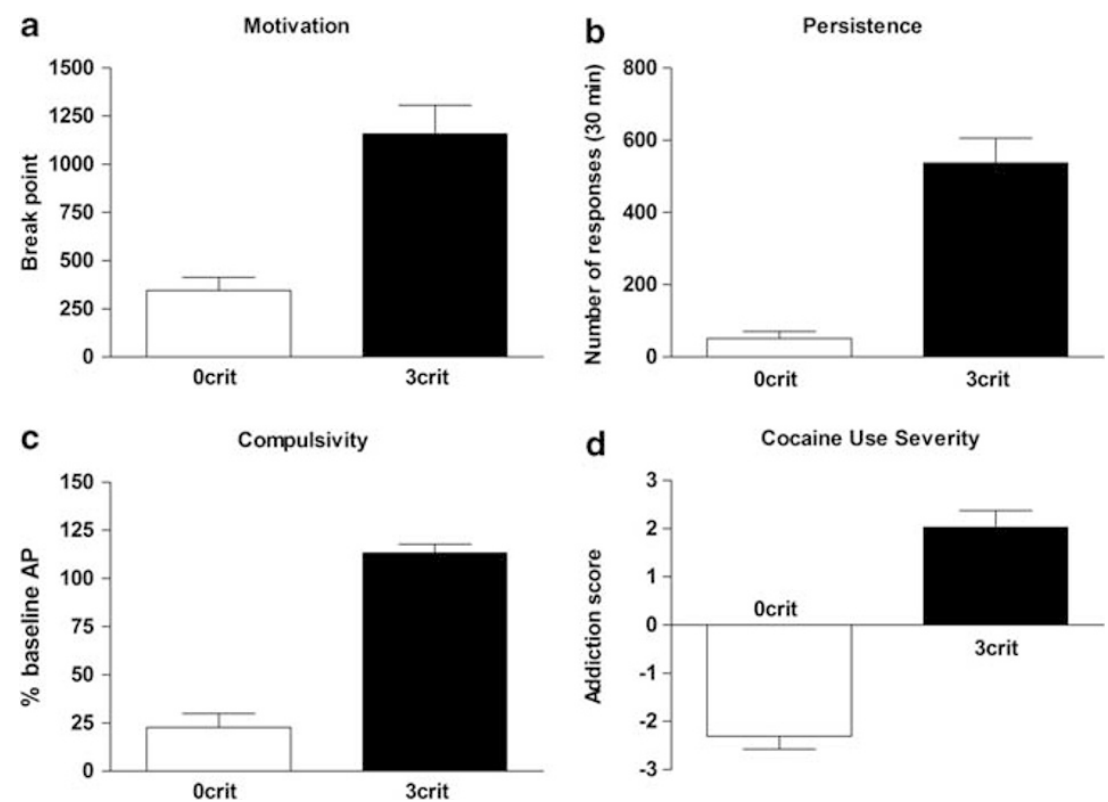

Figure 3 Experiment 2: Rats characterized as addict-like (3crit, $n=5)$ showed higher responses than non-addict-like animals (0crit, $n=7)$ in each criterion for addiction-like behavior. (a) Motivation for cocaine intake expressed by the break point during a progressive ratio session. (b) Persistence of cocaineseeking expressed by active nose-pokes when the drug is signaled as unavailable. (c) Resistance to punishment expressed by \% of baseline active pokes (AP) when cocaine-seeking and taking are paired with a foot-shock punishment ( $0.2 \mathrm{~mA})$. (d) Addict-like rats had a higher cocaine-use severity as measured by computation of the three criteria in a single addiction score scale. Data are presented as mean \pm SEM.

$(\mathrm{F}(1,7)=64.17 ; p<0.0001)$, and an overall interaction of the three factors $(\mathrm{F}(1,7)=12.89 ; p<0.01)$; post hoc analysis confirmed no differences in active and inactive pokes and between groups during extinction. Presentation of cocaine-associated cues selectively reinstated active poking in both groups ( 0 crit $p<0.05,3$ crit $p<0.001$, vs extinction), and active poking induced by cue presentation was significantly higher in 3 crit as compared with 0 crit rats $(p<0.01)$ (Figure 4a).

After reinstatement testing, we examined mGluR2 and mGluR3 gene expression levels. The expression pattern of mGluR2 and mGluR3 was in agreement with previous findings (Ohishi et al, 1993; Tanabe et al, 1993), and showed the highest expression levels for both genes in the cortex and dentate gyrus of the hippocampus (Supplementary Figure S10). No significant differences in mGluR2 and mGluR3 expression were found between 0 crit and 3 crit rats in any investigated forebrain region. For $\mathrm{F}$ - and $p$-values, see Supplementary Table 1 , for mean \pm SEM values in $\mathrm{nCi} / \mathrm{g}$, see Figure $4 \mathrm{~b}$.

Experiment 3: Cocaine Self-Administration, Extinction, and Cue-Induced Reinstatement in mGluR3 - I- and Control Mice

Before self-administration, lever training for food did not significantly differ between the two genotypes tested (data not shown). Acquisition of cocaine $(0.75 \mathrm{mg} / \mathrm{kg} /$ infusion $)$ self-administration measured over the first eight sessions also did not differ significantly between the two genotypes (Figure 5a). ANOVA on the number of infusions revealed no significant effect of genotype $(\mathrm{F}(1,16)=1.72 ; p>0.05)$ or session $\times$ genotype interaction $(\mathrm{F}(7,112)=0.65 ; p>0.05)$. Similarly, no differences were found on the number of lever presses (Supplementary Figure S11A). In a second phase, extinction and reinstatement of cocaine-seeking (Figure 5b) were measured following stable cocaine self-administration. Analysis of the number of lever presses for the last 3 days of cocaine self-administration followed by the extinction and reinstatement sessions revealed no significant effect of genotype $(\mathrm{F}(1,15)=1.34 ; p>0.05)$ or session $\times$ lever $\times$ genotype interaction $(\mathrm{F}(16,240)=0.39 ; p>0.05)$. These results demonstrate that removal of mGluR3 did not affect cue-induced reinstatement.

\section{Experiment 4: Escalation of Cocaine Self-Administration in mGluR3 - / - and Control Mice}

During long access to cocaine self-administration $(0.5 \mathrm{mg} / \mathrm{kg} /$ infusion; 6-h daily sessions) without prior food training, mice from both genotypes rapidly self-administered an increasing amount of cocaine over sessions (Figure 5c). Control and mGluR3 - / - mice displayed similar self-administration over the first 10 sessions (with a maximum of 100 infusions), as well as during the last session, when mice were able to self-administer up to 150 infusions. ANOVA on the number of cocaine infusions showed a significant effect of session $(\mathrm{F}(10,100)=19.1 ; p<0.001)$ that indicates a session-dependent increase in drug-taking in mice from both genotypes. However, the effect of genotype and the genotype $\times$ session interaction was not significant $(\mathrm{F}(1,10)=0.22 ; \quad p>0.05$ and $\mathrm{F}(10,100)=0.8 ; \quad p>0.05$, respectively). Lever presses showed a pattern similar to infusions (Supplementary Figure S11B). These results indicate that the deletion of mGluR3 does not affect the 'escalation-like' pattern of self-administration seen in control mice when given long access to cocaine. 
a Cumulative cue-induced reinstatement
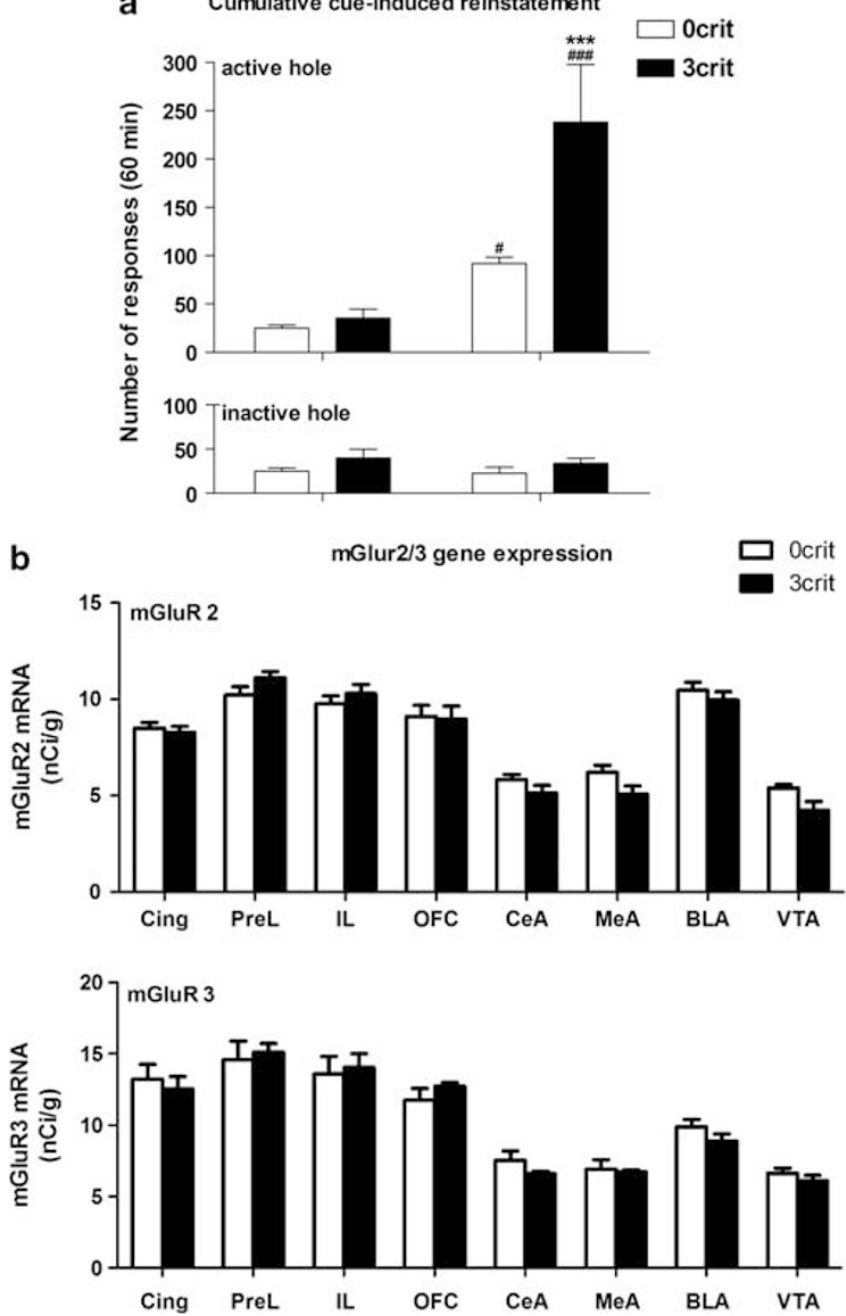

Figure 4 Experiment 2: mGluR2 and mGluR3 mRNA expression levels are not altered in cocaine-addict-like rats. (a) Cumulative cue-induced reinstatement in non-addict-like (0crit) and addict-like (3crit, $n=3$ ) rats. The two groups had a similar level of extinction and reinstated cocaineseeking behavior. Addict-like rats (3crit) had a higher cue-induced reinstatement than non-addict-like rats (0crit, $n=6$ ). (b) Bar graph illustrating mGluR2 (top) and mGluR3 (bottom) mRNA expression in different reward-related forebrain regions of 0 crit and 3 crit rats. Data are expressed as mean $\pm \mathrm{SEM}$ in $\mathrm{nCi} / g$. ${ }^{*} p<0.05$ and $\# \# \#<0.001$ vs extinction; **** $p<0.00$ I vs Ocrit. Abbreviations: Cing, cingulate cortex; PreL, prelimbic cortex; IL, infralimbic cortex; OFC, orbitofrontal cortex; CeA, central amygdala; MeA, medial amygdala; BLA, basolateral amygdala; VTA, ventral tegmental area.

\section{DISCUSSION}

The results reported here demonstrate that stimulation of mGluR2/3 is able to prevent excessive reinstatement of cocaine-seeking in addict-like rats. We replicated previous findings showing that an identical history of cocaine selfadministration yields at least two different, behavioral phenotypes, namely rats without addicted-like behavior (non-addict-like - 0crit) and rats with addict-like behavior (3crit) (Deroche-Gamonet et al, 2004). In comparison with 0 crit, 3 crit rats showed an exacerbated reinstatement of cocaine-seeking and pretreatment with LY379268 abolished cocaine-seeking in both groups. In a previous report by
Kasanetz et al (2012), decreased prefrontal expression of mGluR2/3 and impairment of mGluR2/3-mediated LTD were found in addict-like rats, supporting the hypothesis that addiction-like behavior is maintained by a downregulation of mGluR2/3 functions (see also Meinhardt et al, 2013). In line with Kasanetz and co-worker, we found that the dose of LY379268 necessary to prevent reinstatement in addict-like rats is 10-fold higher than that necessary in nonaddict-like (Figure 2a). In addition, an analysis of patterns of responses suggested that, though higher than in $0 \mathrm{crit}$, in 3 crit rats the effective dose of LY379268 has a longer lasting effect (Figure 2b). To dissect the role of mGluR2 and mGluR3 in mediating cocaine addiction-like behavior, we examined gene expression patterns of both genes in brain sites known to be involved in mediating chronic drug effects (Koob et al, 2010; Noori et al, 2012). After protracted abstinence (37-day withdrawal), no differences in mGluR2 and mGluR3 mRNA between 0 crit and 3 crit rats were found in any analyzed region. A decrease in mGluR $2 / 3$ proteins has been recently reported in the prefrontal cortex of cocaine-addict-like animals (Kasanetz et al, 2012). Although Kazanetz and co-authors studied mGluR2/3 proteins at an earlier time point (24-h withdrawal), the lack of effects at the transcriptional level may indicate a translational regulation of mGluR2/3 in cocaine addiction. In a further attempt to dissect the role of these two receptors, we used knockout mice in the reinstatement and drug escalation model. Since mGluR2 $-/-$ cannot be used in operant procedures due to motoric impairment (Morishima et al, 2005), we only tested mGluR3 - / . mGluR3 - / - mice did not show differences from controls in cocaine self-administration, cocaine escalation, extinction of cocaine-seeking, or cue-induced reinstatement. Together with our finding of unchanged mGluR3 expression in addict-like rats, our data suggest that mGluR2 function is critically involved as a negative feedback mechanism in regulating cue-induced reinstatement, and specific mGluR2 agonists or potentiators are candidate compounds for clinical testing.

Currently, there are no selective agonists or antagonists available to pharmacologically dissect mGluR2 and mGluR3 function. Thus, our mice experiments represent a first step toward the dissection between mGluR2 and mGluR3 functions in reward processing. Earlier rat experiments using the same behavioral models that we used with mGluR3 - / - mice reported that LY379268 may block motivation for cocaine in escalated rats (Hao et al, 2010) and decreased cocaine self-administration and cue-induced reinstatement in short-access cocaine-experienced rats (Baptista et al, 2004; Lu et al, 2012). Since LY379268 does not differentiate between mGluR2 and mGluR3, our mice experiments suggest a prominent role of mGluR2 in cocaine-seeking behavior. In line with this view, it has recently been demonstrated that the mGluR2-positive allosteric modulator BINA decreases cocaine self-administration, cue-induced reinstatement of cocaine-seeking, and the rewarding effects of cocaine, while being devoid of effects on food-driven behavior. In contrast, LY379268 had similar effects in cocaine and food-directed behavior (Jin et al, 2010). Furthermore, several papers have reported that LY379268 treatment inhibits both drug and natural reward-seeking (Baptista et al, 2004; Lu et al, 2007; Peters and Kalivas, 2006; Uejima et al, 2007). These studies suggest 
a

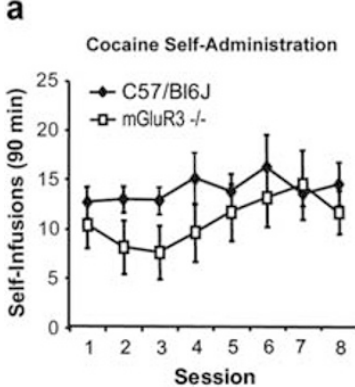

b

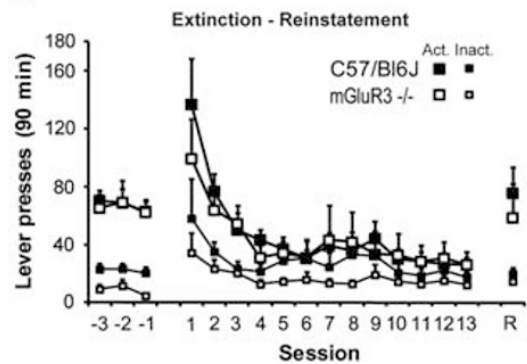

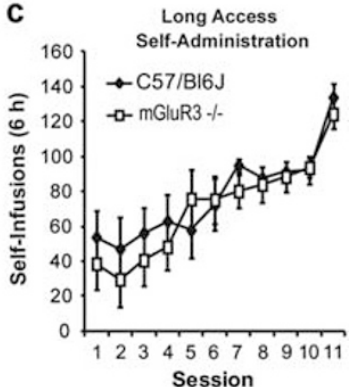

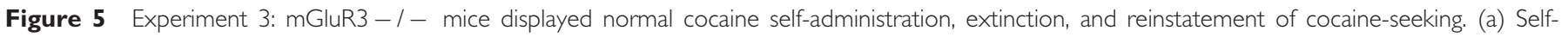

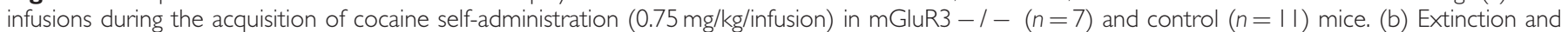

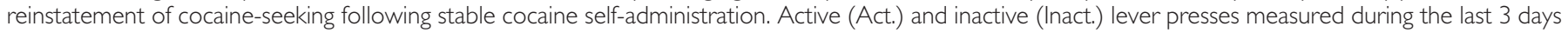

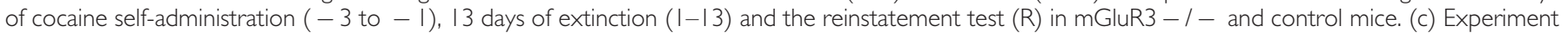

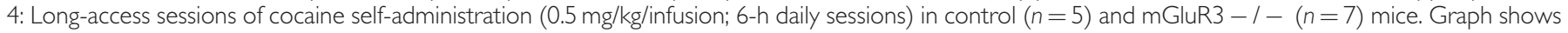

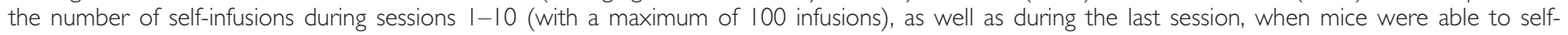
administer up to 150 infusions.

that both mGluR2 and mGluR3 are involved in processing reward-directed behavior, while our data imply that mGluR2 has the primary critical role, at least with respect to cocaineseeking behavior. However, data collected until now suggest that to avoid unwanted side effects, cocaine addiction treatment targeting metabotropic group II glutamate receptors should utilize mGluR2-selective compounds.

The potential validity of $\mathrm{mGluR} 2 / 3$ as targets to treat relapse induced by drug conditioned stimuli may cover several addictive behaviors. In fact, beside cocaine, LY379268 was efficacious in blocking cue- and contextinduced reinstatement of alcohol, heroin, methamphetamine, nicotine, and natural reward-seeking (Bossert et al, 2004, 2005, 2006a, b; Kufahl et al, 2011, 2013; Liechti et al, 2007; Zhao et al, 2006). This wide spectrum ranging from addictive to non-addictive rewards may suggest a more general role for mGluR2/3 in the conditioned effect of drugs. In support with this assumption, a recent paper demonstrated that the increase in dopamine concentration in the NAcs shell induced by a priming injection of nicotine could be blocked by LY379268 when the priming dose was injected in a nicotine-associated context, but not in a neutral context (D’Souza et al, 2011). However, LY379268 can also reduce the primary reinforcing effect of ethanol (Sidhpura et al, 2010), cocaine (Baptista et al, 2004), and nicotine (Liechti et al, 2007), and thus future studies should be addressed to differentiate the role of mGluR2/3 in conditioned and unconditioned effects of drugs.

Although LY379268 has previously been shown to be effective in dampening the reinstatement of cocaine-seeking induced by cues, stress, priming, and the incubation of cocaine-craving in rats (Baptista et al, 2004; Lu et al, 2007; Martin-Fardon and Weiss, 2012; Peters and Kalivas, 2006), this is the first report showing its effectiveness in an animal model of cocaine addiction. Escalation of cocaine-seeking in rats has also been proposed to model some aspects of addiction (Ahmed and Koob, 1998; Lenoir et al, 2012), and a previous report reported that LY379268 blunted motivation for cocaine more efficaciously (ie, at lower dose) in long-access cocaine-escalated rats than in short-access rats (Hao et al, 2010). Furthermore, rats trained with a longaccess cocaine method showed a stable, elevated anxietylike behavior up to 42 days after discontinuation of cocaine- taking that was higher than in short-access animals; importantly, long-access rats were more sensitive to the anxiolytic effect of LY379268 than short-access rats (Aujla et al, 2008). These results are consistent with our report that $3.0 \mathrm{mg} / \mathrm{kg}$ of LY379268 seems to have a longer lasting effect in addict-like than in non-addict-like rats in a cuereinstatement session (Figure $2 \mathrm{~b}$ ). However, in comparison with the cocaine escalation procedure, it is important to consider that the results presented here, as well as those in previous papers, demonstrated that a higher degree of drug exposure is not necessary (Supplementary Figures S4 and S9; Belin et al, 2008, 2009; Deroche-Gamonet et al, 2004) or sufficient (Pelloux et al, 2007) to develop addiction-like behavior. In fact, it was reported that even after 72 days of cocaine self-administration, about $80 \%$ of long-access cocaine-escalated rats still do not differ from short-term access rats in compulsive behavior (Pelloux et al, 2007). Therefore, testing the effects of LY379268 in addict-like and non-addict-like groups of rats may provide more suitable information for the direct translation into the clinical situation. However, we do not propose that the DSM-IVbased rat model of cocaine addiction used here can be routinely used for preclinical compound screening. The procedure is extremely time-consuming and labor- and cost-intensive. We propose that if other animal models, such as the reinstatement model of cocaine-seeking behavior, provide promising initial results with a novel putative anti-relapse compound, the use of the DSM-IVbased rat model would be advisable before clinical testing.

In conclusion, we have demonstrated that rats diagnosed with features of cocaine addiction are sensitive to the anti-relapse properties of LY379268, and we propose that the DSM-IV-based rat model of cocaine addiction is an important final step in the preclinical screening procedure of pharmacological candidates for addiction treatment.

\section{ACKNOWLEDGEMENTS}

We would like to thank Dr Rick Bernardi for assisting with English editing. A special acknowledgement goes to Jean-Francois Fiancette, Prisca Renault, and Eric Balado for training NC. in Bordeaux. This work was supported by 
the Deutsche Forschungsgemeinschaft (DFG): ReinhartKoselleck Award SP 383/5-1 and HA 6102/1-1.

\section{DISCLOSURE}

CC and MC were employed at Glaxo SmithKline and are currently employed at Aptuit. Over the past 3 years, RS has received compensations for research and consultant contracts from Abbott, AirLiquide, GSK, Lundbeck, Reckitt-Benckiser Pharmaceuticals, Roche, and Xenoport.

\section{Authors Contributions}

NC established the 'DSM-IV Based Rat Model of Cocaine Addiction' at the Central Institute of Mental Health in Mannheim (Germany), performed LY379268 experiments, analyzed the data, coordinated the project, and wrote the manuscript; $\mathrm{BH}$ performed mice experiments and analyzed the data; SU and ACH performed in situ hybridization and analyzed the data; LE assisted NC in rat behavioral experiments; $\mathrm{MC}$ and $\mathrm{CC}$ provided mGluR3 - / - mice; VDG trained NC in her laboratory for 5 weeks, gave troubleshooting feedbacks during the establishment of the rat model in Mannheim, and contributed to the revision process; RS designed, supervised, supported the project and wrote the manuscript.

\section{REFERENCES}

Ahmed SH, Koob GF (1998). Transition from moderate to excessive drug intake: change in hedonic set point. Science 282: 298-300.

Aujla H, Martin-Fardon R, Weiss F (2008). Rats with extended access to cocaine exhibit increased stress reactivity and sensitivity to the anxiolytic-like effects of the mGluR 2/3 agonist LY379268 during abstinence. Neuropsychopharmacology 33: 1818-1826.

Baker DA, McFarland K, Lake RW, Shen H, Tang XC, Toda S et al (2003). Neuroadaptations in cystine-glutamate exchange underlie cocaine relapse. Nat Neurosci 6: 743-749.

Baptista MA, Martin-Fardon R, Weiss F (2004). Preferential effects of the metabotropic glutamate $2 / 3$ receptor agonist LY379268 on conditioned reinstatement versus primary reinforcement: comparison between cocaine and a potent conventional reinforcer. J Neurosci 24: 4723-4727.

Belin D, Balado E, Piazza PV, Deroche-Gamonet V (2009). Pattern of intake and drug craving predict the development of cocaine addiction-like behavior in rats. Biol Psychiatry 65: 863-868.

Belin D, Mar AC, Dalley JW, Robbins TW, Everitt BJ (2008). High impulsivity predicts the switch to compulsive cocaine-taking. Science 320: 1352-1355.

Bossert JM, Busch RF, Gray SM (2005). The novel mGluR2/3 agonist LY379268 attenuates cue-induced reinstatement of heroin seeking. Neuroreport 16: 1013-1016.

Bossert JM, Gray SM, Lu L, Shaham Y (2006a). Activation of group II metabotropic glutamate receptors in the nucleus accumbens shell attenuates context-induced relapse to heroin seeking. Neuropsychopharmacology 31: 2197-2209.

Bossert JM, Liu SY, Lu L, Shaham Y (2004). A role of ventral tegmental area glutamate in contextual cue-induced relapse to heroin seeking. J Neurosci 24: 10726-10730.

Bossert JM, Poles GC, Sheffler-Collins SI, Ghitza UE (2006b). The mGluR2/3 agonist LY379268 attenuates context- and discrete cue-induced reinstatement of sucrose seeking but not sucrose self-administration in rats. Behav Brain Res 173: 148-152.
Corti C, Battaglia G, Molinaro G, Riozzi B, Pittaluga A, Corsi M et al (2007). The use of knock-out mice unravels distinct roles for mGlu2 and mGlu3 metabotropic glutamate receptors in mechanisms of neurodegeneration/neuroprotection. J Neurosci 27: 8297-8308.

D'Souza MS, Liechti ME, Ramirez-Nino AM, Kuczenski R, Markou A (2011). The metabotropic glutamate $2 / 3$ receptor agonist LY379268 blocked nicotine-induced increases in nucleus accumbens shell dopamine only in the presence of a nicotineassociated context in rats. Neuropsychopharmacology 36: 2111-2124.

Deroche-Gamonet V, Belin D, Piazza PV (2004). Evidence for addiction-like behavior in the rat. Science 305: 1014-1017.

Hansson AC, Sommer W, Rimondini R, Andbjer B, Stromberg I, Fuxe K (2003). c-fos reduces corticosterone-mediated effects on neurotrophic factor expression in the rat hippocampal CA1 region. J Neurosci 23: 6013-6022.

Hao Y, Martin-Fardon R, Weiss F (2010). Behavioral and functional evidence of metabotropic glutamate receptor $2 / 3$ and metabotropic glutamate receptor 5 dysregulation in cocaineescalated rats: factor in the transition to dependence. Biol Psychiatry 68: 240-248.

Jin X, Semenova S, Yang L, Ardecky R, Sheffler DJ, Dahl R et al (2010). The mGluR2 positive allosteric modulator BINA decreases cocaine self-administration and cue-induced cocaineseeking and counteracts cocaine-induced enhancement of brain reward function in rats. Neuropsychopharmacology 35: 2021-2036.

Kalivas PW (2009). The glutamate homeostasis hypothesis of addiction. Nat Rev Neurosci 10: 561-572.

Kalivas PW, Volkow ND (2005). The neural basis of addiction: a pathology of motivation and choice. Am J Psychiatry 162: 1403-1413.

Kasanetz F, Deroche-Gamonet V, Berson N, Balado E, Lafourcade M, Manzoni O et al (2010). Transition to addiction is associated with a persistent impairment in synaptic plasticity. Science 328: 1709-1712.

Kasanetz F, Lafourcade M, Deroche-Gamonet V, Revest JM, Berson N, Balado E et al (2012). Prefrontal synaptic markers of cocaine addiction-like behavior in rats. Mol Psychiatry; doi:10.1038/ mp.2012.59 (e-pub ahead of print).

Koob GF, Volkow ND (2010). Neurocircuitry of addiction. Neuropsychopharmacology 35: 217-238.

Kufahl PR, Martin-Fardon R, Weiss F (2011). Enhanced sensitivity to attenuation of conditioned reinstatement by the mGluR $2 / 3$ agonist LY379268 and increased functional activity of mGluR 2/3 in rats with a history of ethanol dependence. Neuropsychopharmacology 36: 2762-2773.

Kufahl PR, Watterson LR, Nemirovsky NE, Hood LE, Villa A, Halstengard C et al (2013). Attenuation of methamphetamine seeking by the mGluR2/3 agonist LY379268 in rats with histories of restricted and escalated self-administration. Neuropharmacology 66: 290-301.

Lenoir M, Guillem K, Koob GF, Ahmed SH (2012). Drug specificity in extended access cocaine and heroin self-administration. Addict Biol 17: 964-976.

Liechti ME, Lhuillier L, Kaupmann K, Markou A (2007). Metabotropic glutamate $2 / 3$ receptors in the ventral tegmental area and the nucleus accumbens shell are involved in behaviors relating to nicotine dependence. J Neurosci 27: 9077-9085.

Lu L, Uejima JL, Gray SM, Bossert JM, Shaham Y (2007). Systemic and central amygdala injections of the mGluR(2/3) agonist LY379268 attenuate the expression of incubation of cocaine craving. Biol Psychiatry 61: 591-598.

Lu L, Xue Y, Steketee JD, Rebec GV, Sun W (2012). Regulation of cocaine-induced reinstatement by group II metabotropic glutamate receptors in the ventral tegmental area. Psychopharmacology (Berl) 220: 75-85. 
Martin-Fardon R, Weiss F (2012). (-)-2-oxa-4-aminobicylco[3.1.0]hexane-4,6-dicarboxylic acid (LY379268) and 3-[(2-methyl-1, 3-thiazol-4-yl)ethynyl]piperidine (MTEP) similarly attenuate stress-induced reinstatement of cocaine seeking. Addict Biol 17: 557-564.

Meinhardt MW, Hansson AC, Perreau-Lenz S, Bauder-Wenz C, Stahlin O, Heilig M et al (2013). Rescue of infralimbic mGluR2 deficit restores control over drug-seeking behavior in alcohol dependence. J Neurosci 33: 2794-2806.

Morishima Y, Miyakawa T, Furuyashiki T, Tanaka Y, Mizuma H, Nakanishi S (2005). Enhanced cocaine responsiveness and impaired motor coordination in metabotropic glutamate receptor subtype 2 knockout mice. Proc Natl Acad Sci USA 102: $4170-4175$.

Moussawi K, Zhou W, Shen H, Reichel CM, See RE, Carr DB et al (2011). Reversing cocaine-induced synaptic potentiation provides enduring protection from relapse. Proc Natl Acad Sci USA 108: 385-390.

Noori HR, Spanagel R, Hansson AC (2012). Neurocircuitry for modeling drug effects. Addict Biol 17: 827-864.

Ohishi H, Shigemoto R, Nakanishi S, Mizuno N (1993). Distribution of the messenger RNA for a metabotropic glutamate receptor, mGluR2, in the central nervous system of the rat. Neuroscience 53: 1009-1018.

Pelloux Y, Everitt BJ, Dickinson A (2007). Compulsive drug seeking by rats under punishment: effects of drug taking history. Psychopharmacology (Berl) 194: 127-137.

Peters J, Kalivas PW (2006). The group II metabotropic glutamate receptor agonist, LY379268, inhibits both cocaine- and food-seeking behavior in rats. Psychopharmacology (Berl) 186: 143-149.

Sanchis-Segura C, Spanagel R (2006). Behavioural assessment of drug reinforcement and addictive features in rodents: an overview. Addict Biol 11: 2-38.

Sidhpura N, Weiss F, Martin-Fardon R (2010). Effects of the mGlu2/3 agonist LY379268 and the mGlu5 antagonist MTEP on ethanol seeking and reinforcement are differentially altered in rats with a history of ethanol dependence. Biol Psychiatry 67: 804-811.

Tanabe Y, Nomura A, Masu M, Shigemoto R, Mizuno N, Nakanishi S (1993). Signal transduction, pharmacological properties, and expression patterns of two rat metabotropic glutamate receptors, mGluR3 and mGluR4. J Neurosci 13: 1372-1378.

Uejima JL, Bossert JM, Poles GC, Lu L (2007). Systemic and central amygdala injections of the mGluR2/3 agonist LY379268 attenuate the expression of incubation of sucrose craving in rats. Behav Brain Res 181: 292-296.

Wikler A, Pescor FT (1967). Classical conditioning of a morphine abstinence phenomenon, reinforcement of opioid-drinking behavior and 'relapse' in morphine-addicted rats. Psychopharmacologia 10: 255-284.

Wolffgramm J, Heyne A (1995). From controlled drug intake to loss of control: the irreversible development of drug addiction in the rat. Behav Brain Res 70: 77-94.

Zhao Y, Dayas CV, Aujla H, Baptista MA, Martin-Fardon R, Weiss F (2006). Activation of group II metabotropic glutamate receptors attenuates both stress and cue-induced ethanolseeking and modulates c-fos expression in the hippocampus and amygdala. J Neurosci 26: 9967-9974.

Supplementary Information accompanies the paper on the Neuropsychopharmacology website (http://www.nature.com/npp) 